\section{MS22-04 Experimental Electron Density of Low-Barrier Hydrogen Bonds in $\mathrm{H}_{3} \mathrm{Co}(\mathrm{CN})_{6}$ \\ Kasper Tolborg ${ }^{1}$, Bo B. Iversen ${ }^{1}$}

1. Center for Materials Crystallograhy, Department of Chemistry, Aarhus University

email: tolborg@chem.au.dk

Hydrogen bonding is the most important directional intermolecular interaction with importance in a wide range of fields across natural science. Electron density studies may quantify hydrogen bonding behavior in compounds with very strong hydrogen bonds and explain their importance for particular properties.

Recent PDF studies on $\mathrm{H}_{3} \mathrm{Co}(\mathrm{CN})_{6}$, which has one of the shortest N-H-N hydrogen bonds known in the literature, has shown a significant effect of isotope substitution on the negative thermal expansion (NTE) behavior of the crystals, i.e. the protonated compound has a significantly larger NTE than the deuterated compound [1]. In this study, the experimental electron density distribution in $\mathrm{H}_{3} \mathrm{Co}(\mathrm{CN})_{6}$ is evaluated using synchrotron single crystal X-ray diffraction and neutron powder diffraction.

Single crystal X-ray diffraction were measured at BL02B1, Spring8, Japan on both protonated and deuterated samples at $20 \mathrm{~K}$ and $100 \mathrm{~K}$ with resolutions up to $1.67 \AA^{-1}$. These are combined with powder neutron diffraction data to get a better description of the hydrogen position and thermal parameters.

The X-ray data have been modelled with the extended Hansen-Coppens multipole formalism [2] and the resulting density is analyzed by the Quantum Theory of Atoms in Molecules. The Co-CN interaction is found to be of intermediate character, with very similar properties as those reported for transition metal carbonyls in the literature [3]. This is interesting, since the formal charges are very different in two cases.

The properties along the bond path for the hydrogen bond is very dependent on the position of hydrogen. A symmetric arrangement as proposed for hydrogen at low temperature, gives two shared interactions very similar to the ones seen for the $\mathrm{O}-\mathrm{H}-\mathrm{O}$ low-barrier hydrogen bond in Benzoylacetone [4], explaining the origin of the negative thermal expansion as arising from a covalently bonded network. An asymmetric arrangement gives more shared character to the donor atom bonding and more closed shell character to the acceptor atom, making the crystal more molecular of nature.

1. Keen, D. A., et al., J. Phys.: Condens. Matter 2010, 22, 404202-404208

2. Fischer, A. et al., J. Phys. Chem. A 2011, 115, 13061-13071

3. Farrugia et al., J. Phys. Chem. A 2005, 109, 8834-8848

4. Madsen et al., J. Am. Chem. Soc. 1998, 120, 10040-10045

Keywords: Hydrogen Bonds, Electron Density, Negative Thermal Expansion

\section{MS22-05 Electrostatic Potential of Dynamic Charge Densities}

\author{
Christian B. Hübschle ${ }^{1}$, Sander van Smaalen ${ }^{1}$
}

1. Laboratory of Crystallography, University of Bayreuth

email: chuebsch@moliso.de

The computer program PRIOR, which is part of the BayMEM suite [1] is able to calculate a dynamic electron density from a multipole model [2]. Such a density or the result of a maximum entropy method (MEM) calculation can be used to calculate a electrostatic potential by a modified version of the PRIOR program.

The idea of the algorithm is based on the reciprocal part of an Ewald summation [3]. The application of the $U_{i j}$ parameter to the nuclear density in the above equation improves the convergence of the summation so that a calculation in direct space is unnecessary, which make this approach quite fast.

We will show applications to boron and boron rich compounds.

[1] L. Palatinus, S. van Smaalen and M. Schneider. Acta Crystallogr. (2003) A59 459-469.

[2] S. Mondal, S. J. Prathapa and S. van Smaalen. Acta Crystallogr. (2012) A68 568-581.

[3] P. Ewald (1921). "Die Berechnung optischer und elektrostatischer gitterpotentiale." Annals Phys. 64, 253-287.

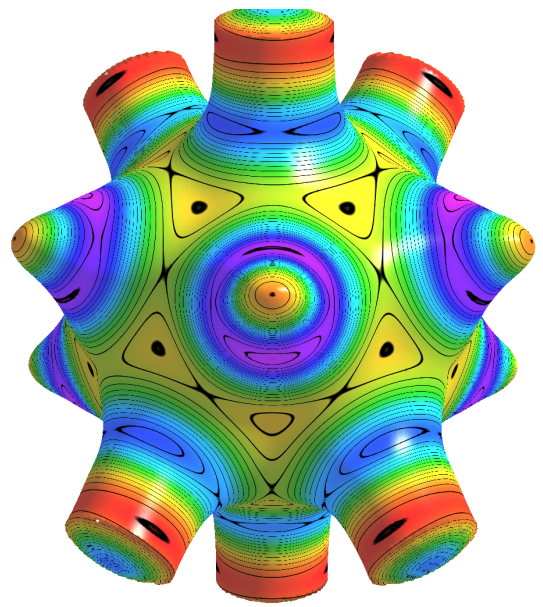

Figure 1. Electrostatic potential of the dynamic charge density of $\alpha$-boron mapped on an iso density surface.

Keywords: electrostatic potential, boron, dynamic density 